\title{
Estructura y composición florística del bosque seco tropical de Sanguaré-Sucre (Colombia)
}

\author{
Structure and Floristic Composition of Tropical Dry Forest of Sanguaré-Sucre \\ (Colombia)
}

\author{
Danny Rafael Sanmartín-Sierra ${ }^{\mathrm{a}}$ \\ Diego Fernando Angarita-Hernández ${ }^{\mathrm{a}}$ \\ Jorge D. Mercado-Gómez ${ }^{\mathrm{b}^{*}}$
}

Recepción: 25-ene-2016

Aceptación: 07-may-2016

\section{Resumen}

En el presente estudio se analizó la estructura y composición de la vegetación en dos fragmentos de bosque seco tropical en la Reserva Natural Sanguaré (San Onofre, Sucre, Colombia). Se estableció la riqueza de plantas y ciertos parámetros estructurales para medir la organización y dinámica del bosque. En cada fragmento de bosque se hicieron levantamientos de vegetación con el método de transectos tipo RAP modificados. Se realizaron identificaciones, conteos de especies y mediciones de estructura como, la altura y el DAP, además se establecieron las familias, géneros y especies más importantes; los hábitos de crecimiento y el perfil de vegetación. Se encontraron 658 individuos, agrupados en 37 familias, 81 géneros y 82 especies. Los grupos taxonómicos más importantes fueron Fabaceae, Malvaceae, Mimosaceae, Caesalpinaceae, Rubiaceae y Moraceae en términos de riqueza; mientras que grupos abundantes como Sapindaceae, Malvaceae, Fabaceae, Bignoniaceae, Rubiaceae y Apocynaceae sobresalieron. En el hábito de crecimiento, los árboles fueron dominantes y el palmetum tuvo una alta representación, como un dato atípico para el bosque seco. La mayor altura de árboles fue de 15 metros en promedio, lo que nos permite inferir que los bosques se encuentran en estado de sucesión temprana.

Palabras clave: Flora, Golfo de Morrosquillo, Riqueza, San Onofre.

\begin{abstract}
In the present study we analyzed the structure and composition of a tropical dry forest fragment in the Natural Reserve Sanguare (San Onofre, Sucre, Colombia). We established the richness of plants and the structural parameters to measure the organization and dynamics of the forest. In each forest fragments we made a vegetation survey with RAP's modified transect type method. We made taxonomic identification, species counts and structure measurements such as height and DBH, to establish the most important families, genera and species; the growth habits and vegetation profile. We found 658 individuals, grouped in 37 families, 81 genera and 82 species. The most important taxonomic in terms of richness were Malvaceae, Fabaceae, Caesalpinaceae, Mimosaceae, Rubiaceae and Moraceae; while as an abundant taxa Caesalpinaceae,

${ }^{a}$ Reserva Natural Sanguaré, San Onofre, Sucre.

${ }^{b}$ Departamento de Biología y Química, Grupo Evolución y Sistemática Tropical. Universidad de Sucre, Sincelejo, Colombia.

*Autor de correspondencia: jorge.mercado@unisucre.edu.co
\end{abstract}




\begin{abstract}
Sapindaceae, Malvaceae, Bignoniaceae y Rubiaceae where important. The dominant habits of growth were trees and the palmetum had a high representation as an outlier to the dry forest. The greatest height of trees was 15 meters on average, which allows us to infer the forest is in an early succession.
\end{abstract}

Key words: Flora, Morrosquillo Gulf, Richness, San Onofre.

\section{Introducción}

El bosque seco tropical (bs-t) es considerado uno de los ecosistemas más complejos e interesantes de la naturaleza en el Neotrópico, producto de las adaptaciones que han sufrido sus especies a las altas temperaturas y escasa disponibilidad del agua $[1,2]$. Se caracteriza por presentar una temperatura superior a los $17^{\circ} \mathrm{C}$, precipitación anual entre 250 a $2000 \mathrm{~mm}$ [3] y una marcada estacionalidad seca. Sin embargo, a pesar de su valor biológico (ecología, evolución, especiación) en el Neotrópico, este ecosistema es uno de los más amenazados, producto de su larga historia de transformación, consecuencia del avance antrópico en agricultura y ganadería [4].

En Colombia estos bosques están distribuidos principalmente sobre los valles interandinos y en la región Caribe; sobre los valles intermedios la mayor extensión se observa en el valle del río Magdalena, y en pequeños remanentes aislados del valle del río Cauca [5]; mientras que en el Caribe, en los Montes de María, el Parque Tayrona, Tierra Bomba, entre otros [6]. Al igual que en el resto del Neotrópico, el uso de sus suelos para agricultura y ganadería, ha llevado a este ecosistema, por ejemplo, en el Caribe a presentar una cobertura que corresponde al $3.2 \%$ (133,133 ha) de su estado original, la cual está representada por solo ocho remanentes, en su mayoría aislados y fragmentados [3]. Asimismo, estos bosques enfrentan no solo una problemática de conservación, también de investigación; ya que pocos han sido los estudios que se han desarrollado sobre las planicies del Caribe para determinar la diversidad existente en estos remantes de bosque. Se incluyen dentro de estos trabajos el de Mendoza [7] quien analiza la flora en varios fragmentos del Caribe y los relaciona florísticamente con sitios del valle del Magdalena. En la Guajira se han elaborado análisis de la estructura de la vegetación de plantas leñosas y un catálogo de las epifitas en la Serranía de la Macuira [8-10]; por su parte, en Magdalena, específicamente sobre el Parque Tayrona [11-13], además de algunos estudios con amplio rango geográfico como los llevados a cabo por Cuadros [14] y el Instituto Alexander von Humboldt $[15,16]$ sobre la llanura del Caribe.
Sin embargo, existen varios fragmentos de bosque en algunos departamentos que aún no han sido estudiados. Tal es el caso del departamento de Sucre, en el cual la flora tiene un conocimiento deficiente y los trabajos abordados se han enfocado en plantas medicinales [17] y en estudios florísticos en la Reserva Primates en los Montes de María [3]. En este sentido, Cuervo et al. [18] y Galván-Guevara et al. [19] han analizado la diversidad y composición de la flora; mientras que Patiño y Rangel [20] llevaron a cabo un estudio fitosociológico y Gentry [21] realizó algunas observaciones sobre la flora, hallando el registro de Buxus citrifolia para el Caribe. Adicionalmente, en otras localidades de Montes de María, Sampedro, et al. [21] analiza el estado de la vegetación en sitios abandonados por el desplazamiento; mientras que Sampedro, et al. [23] establece las especies que son promisorias para el ecoturismo y algunos análisis de la estructura de la vegetación en Tolúviejo [24-27].

En la subregión Golfo de Morrosquillo, poco ha sido el estudio de la flora, teniendo en cuenta que existen extensos fragmentos de bosque seco vinculados a ecosistemas de manglar [28] y otros fragmentos aislados que se encuentran en algún estado de sucesión [29, 30], los cuales poseen la mayor extensión en esta subregión y son importantes para el ecoturismo y el mantenimiento de los procesos ecológicos. Parte de estos fragmentos se encuentran ubicados en la Reserva Sanguaré en el municipio de San Onofre (Sucre, Colombia). De estos dos fragmentos solo se tiene hasta la fecha un reporte corológico [29], y se desconoce la estructura de la vegetación. En el presente estudio se analizó la estructura, riqueza y composición de flora leñosa con base en un muestreo tipo RAP modificado, sobre dos puntos en la Reserva Natural Sanguaré, además se realizaron comparaciones con otros fragmentos de bosque seco en la región Caribe.

\section{Metodología}

\section{1. Área de estudio}

La Reserva Natural de la Sociedad Civil Sanguaré se encuentra ubicada en la punta norte del 
Golfo de Morrosquillo en el municipio de San Onofre, departamento de Sucre, sobre los 9 ${ }^{\circ} 42^{\prime} 37.30^{\prime \prime} \mathrm{N}$ y $75^{\circ} 40^{\prime} 59.75$ 'O (figura 1 ). El clima se caracteriza por ser seco con una temperatura que varía entre los 38 a $24^{\circ} \mathrm{C}$ durante el año y la precipitación se encuentra entre los 800 y $1,000 \mathrm{~mm}$ anuales con la formación de dos períodos secos en Diciembre a Abril y Junio a Julio, en los meses restantes hay lluvias [28]. En este territorio se localiza un enclave de bosque seco tropical y porciones de sabanas antrópicas de lomerío y de montaña [30]; además, en el litoral costero, existen los ecosistemas de manglar y lagunas costeras [30, 31].

\subsection{Muestreo de la vegetación}

Se realizaron levantamientos de la vegetación en dos enclaves de bosque, el primero con un aérea de 110 ha y el segundo con 23 ha (figura 1). En cada fragmento se utilizaron transectos tipo RAP (Rapid Assessment Program) con 5 sub-parcelas de $50 \mathrm{~m} \times$ $2 \mathrm{~m}$ para cada fragmento [32], con algunas modificaciones propuestas por ISA-JAUM [32], contando y midiendo todos los individuos con diámetro a la altura del pecho (DAP) de $1 \mathrm{~cm}$ de todos los árboles y arbustos excluyendo lianas y hierbas. Adicionalmente y con el fin de ampliar el listado de la vegetación, se llevaron a cabo varios recorridos sobre los llamados "corredores biológicos". De cada una de las especies se recolectaron tres individuos, además de aquellos datos que se pudieran perder en el proceso de prensado y secado (colores, olores, entre otros).

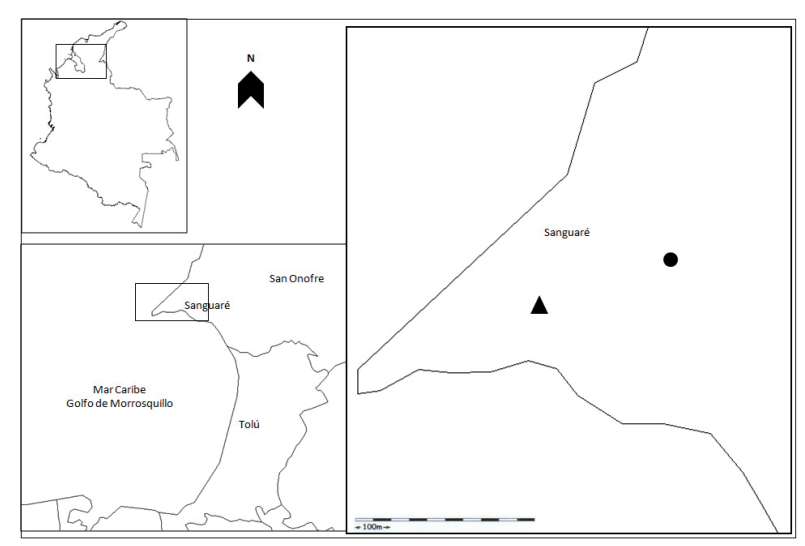

Figura 1. Ubicación geográfica la Reserva Natural de la Sociedad Civil Sanguaré en el municipio de San Onofre (departamento de Sucre, Colombia), en la imagen a gran escala se observan los puntos de muestreo de 110 ha ( $(\mathbf{\Lambda})$ y 23 ha $(\bullet)$.
Para cada individuo se estimó la altura (m) utilizando un nivel abney, se midió el DAP $(\mathrm{cm})$, se enumeró y se registró el geoposicionamiento (GARMIN-ETREX10).

Una vez recolectados los ejemplares, estos fueron prensados y herborizados según los métodos convencionales para tal proceso. Posteriormente, las especies fueron identificadas por medio de dos métodos principalmente. El primero consiste en el uso de claves taxonómicas [34-36] y ayudas en la web (MOBOT Tropics). El segundo realizando comparaciones sobre colecciones, directas de Herbario (HUA) y online (Herbario COL, Herbario del bosque seco tropical). Finalmente, las muestras fueron corroboradas por expertos botánicos en los casos necesarios y su nomenclatura fue revisada en Taxonomic Name Resolution Service v4.0 [37]. Todos los ejemplares se encuentran depositados en el Herbario de la Universidad de Antioquia (HUA).

\subsection{Análisis de la información}

Una vez identificadas todas las especies, estas fueron organizadas con base en el sistema de clasificación de la APG III [38] y al interior de cada taxón, los géneros y especies se organizaron en orden alfabético. Con el fin de analizar la estructura, composición y riqueza, se llevaron a cabo conteos por familias, géneros y especies. Inicialmente se calcularon y graficaron la abundancia por especies e individuos de todas las familias. Seguidamente fueron estimadas la dominancia y frecuencia de todas las especies más importantes, entendiendo a estas como aquellos taxones más representativos en términos de número y distribución de individuos.

Para estimar los patrones de estructura fueron contados y tabulados todos los individuos con DAP $\geq 1 \mathrm{~cm}$ comprendidos por árboles, arbustos y palmas. Asimismo, se analizó y graficó la distribución de clases según la altura. Se crearon intervalos o categorías para analizar los estratos verticales en el bosque de acuerdo a lo establecido por Rangel y Velásquez [39]. Finalmente, para evaluar la importancia de las especies y familias, se construyó el índice de valor de Importancia (IVI), por especies y familia según la metodología propuesta por van der Hammen y Rangel [40]. 


\section{Resultados}

\subsection{Riqueza y composición florística}

Un total de 658 individuos entre árboles y arbusto leñosos, distribuidos en 37 familias, 81 géneros y 82 especies, excluidas hierbas y lianas (tabla 1) fueron determinados en los transectos de muestreo. En el corredor biológico se hallaron 27 individuos di- ferentes de árboles y arbusto leñosos, pertenecientes a 16 familias, 22 géneros y 19 especies (tabla 1). Las familias con mayor abundancia fueron Sapindaceae, Malvaceae, Fabaceae, Bignoniaceae, Rubiaceae y Apocynaceae (figura 2a). Mientras que a nivel de especies fueron relevantes Fabaceae, Malvaceae, Mimosaceae, Caesalpinaceae, Rubiaceae y Moraceae (figura 2b).

Tabla 1. Listado de especies de plantas leñosas encontradas en el bosque (1) y corredores biológicos (2) ubicados en la reserva Sanguaré-Sucre (Colombia).

\begin{tabular}{|c|c|c|}
\hline Familia & Especies & Nombre común \\
\hline Acanthaceae & Aphelandra albert-smithii Leonard $^{\top}$ & Solita \\
\hline Anacardiaceae & Anacardium excelsum (Bertero \& Balb. ex Kunth) Skeels ${ }^{1}$ & Caracolí \\
\hline Anacardiaceae & Astronium graveolens Jacq. ${ }^{1}$ & Santa cruz \\
\hline Anacardiaceae & Spondias mombin $\mathrm{L}^{1}$ & Jobo \\
\hline Apocynaceae & Rauvolfia tetraphylla $\mathrm{L}^{1}$ & Cruceta \\
\hline Apocynaceae & Tabernaemontana cymosa Jacq. ${ }^{1}$ & Lechoso ó Cojón de fraile \\
\hline Apocynaceae & Tabernaemontana grandiflora Jacq. ${ }^{1}$ & \\
\hline Arecaceae & Attalea butyracea (Mutis ex L. f.) Wess. Boer ${ }^{1}$ & Palma de vino \\
\hline Arecaceae & Copernicia tectorum Mart. $^{1}$ & Palma sará \\
\hline Bignoniaceae & Crescentia cujete L. $^{1}$ & Totumo \\
\hline Bignoniaceae & Tabebuia billbergii (Bureau \& K. Schum.) Standl. ${ }^{1}$ & Tropillo \\
\hline Bignoniaceae & Tabebuia ochracea (Cham.) Standl. ${ }^{1}$ & Polvillo \\
\hline Bignoniaceae & Tabebuia rosea (Bertol.) A. DC. ${ }^{1}$ & Roble \\
\hline Boraginaceae & Cordia barbata $\mathrm{J}$. Estrada ${ }^{1}$ & Maíz tostado \\
\hline Boraginaceae & Cordia collococca L. $^{1}$ & Muñeco \\
\hline Burseraceae & Bursera graveolens (Kunth) Triana \& Planch. ${ }^{1}$ y 2 & Capiña o caraña \\
\hline Burseraceae & Bursera simaruba (L.) Sarg. ${ }^{1}$ & Indio encuero \\
\hline Caesalpiniaceae & Caesalpinia pulcherrima (L.) Sw. ${ }^{1}$ y 2 & \\
\hline Caesalpiniaceae & Delonix regia (Bojer ex Hook.) Raf. ${ }^{1}$ y 2 & \\
\hline Caesalpiniaceae & Bauhinia picta (Kunth) DC. ${ }^{1}$ & Clavellina \\
\hline Caesalpiniaceae & Caesalpinia coriaria (Jacq.) Willd. ${ }^{1}$ & Pata vaca \\
\hline Caesalpiniaceae & Copaifera officinalis L. ${ }^{1}$ & Acacio \\
\hline Caesalpiniaceae & Hymenaea courbaril L. ${ }^{1}$ & Cascarilla \\
\hline Caesalpiniaceae & Senna alata (L.) Roxb. ${ }^{1}$ & Orillero \\
\hline Capparaceae & Capparidastrum frondosum (Jacq.) Cornejo \& Iltis $^{1}$ & \\
\hline Capparaceae & Crateva tapia $\mathrm{L} .1$ & Yarumo ó Guarumo \\
\hline Capparaceae & Quadrella odoratissima (Jacq.) Hutch. ${ }^{1 y 2}$ & Árbol de los deseos \\
\hline Celastraceae & Maytenus macrocarpa (Ruiz \& Pav.) Briquet ${ }^{1}$ & \\
\hline Chrysobalanaceae & Chrysobalanus icaco L. ${ }^{1}$ y 2 & Icaco \\
\hline Cochlospermaceae & Cochlospermum vitifolium (Willd.) Spreng. ${ }^{1}$ & Papayote \\
\hline Combretaceae & Conocarpus erectus L. 1 y 2 & Zaragoza \\
\hline Combretaceae & Laguncularia racemosa (L.) C.F. Gaertn. ${ }^{1}$ & Mangle bobo \\
\hline Combretaceae & Terminalia catappa $\mathrm{L}^{1}$ & Almendro \\
\hline Dilleniaceae & Curatella americana L. ${ }^{1}$ y 2 & Peralejo \\
\hline Ebenaceae & Diospyros inconstans Jacq. ${ }^{1}$ & Juan de Dios \\
\hline Erythroxylaceae & Erythroxylum carthagenense Jacq. ${ }^{1}$ & Coca criolla \\
\hline Euphorbiaceae & Croton magdalenensis Müll. Arg. ${ }^{1}$ & \\
\hline Euphorbiaceae & Hura crepitans $\mathrm{L}^{1}$ & Ceiba de leche \\
\hline Euphorbiaceae & Sapium glandulosum (L.) Morong ${ }^{1}$ & Ñipi ñipi \\
\hline Fabaceae & Andira inermis (W. Wright) $\mathrm{DC}^{1}$ & Guama de arrollo \\
\hline Fabaceae & Centrolobium yavizanum Pittier $^{1 y 2}$ & Balaustre \\
\hline Fabaceae & Gliricidia sepium (Jacq.) Kunth ex Walp. ${ }^{1}$ & \\
\hline Fabaceae & Machaerium arboreum (Jacq.) Benth. ${ }^{1}$ & Guayacán de bola \\
\hline Fabaceae & Machaerium capote Triana ex Dugand 1 & Matarratón \\
\hline Fabaceae & Machaerium microphyllum (E. Mey.) Standl. ${ }^{1}$ & Látigo \\
\hline
\end{tabular}


Continuación Tabla 1.

\begin{tabular}{|c|c|c|}
\hline Familia & Especies & Nombre común \\
\hline Fabaceae & Myrospermum frutescens Jacq. ${ }^{1}$ & \\
\hline Fabaceae & Platymiscium pinnatum (Jacq.) Dugand ${ }^{1}$ & Ají de monte \\
\hline Fabaceae & Pterocarpus acapulcensis Rose $^{1}$ & 7 cuero \\
\hline Lauraceae & Nectandra membranácea (Sw.) Griseb. ${ }^{1}$ & Sangregado \\
\hline Lecythidaceae & Lecythis minor Jacq. ${ }^{1}$ & Trébol \\
\hline Malpighiaceae & Byrsonima aerugo Sagot 1 y 2 & Noro \\
\hline Malpighiaceae & Byrsonima crassifolia (L.) Kunth $1 y 2$ & Mantequero \\
\hline Malvaceae & Apeiba tibourbou Aubl. ${ }^{1}$ & Verrugoso \\
\hline Malvaceae & Ceiba pentandra (L.) Gaertn. ${ }^{1}$ y 2 & Ceiba \\
\hline Malvaceae & Guazuma ulmifolia Lam. ${ }^{1}$ & Tallo hueco \\
\hline Malvaceae & Helicteres guazumifolia Kunth $^{1}$ & Suingle \\
\hline Malvaceae & Luehea seemannii Triana \& Planch. ${ }^{1}$ & \\
\hline Malvaceae & Pachira quinata (Jacq.) W.S.Alverson ${ }^{1}$ & Ceiba tolua \\
\hline Malvaceae & Sterculia apetala (Jacq.) H. Karst. ${ }^{1}$ & \\
\hline Malvaceae & Swietenia macrophylla King ${ }^{1}$ & Majagua de gallina \\
\hline Meliaceae & Trichilia hirta L. $^{1}$ & Culi hiero \\
\hline Meliaceae & Trichilia pallida Sw. $^{1}$ & Laurel \\
\hline Mimosaceae & Acacia collinsii Saff. ${ }^{1}$ & Algarrobo \\
\hline Mimosaceae & Acacia mangium Willd. 1 y 2 & \\
\hline Mimosaceae & Albizia niopoides (Spruce ex Benth.) Burkart ${ }^{1}$ & Bajagua \\
\hline Mimosaceae & Calliandra riparia Pittier $1{ }^{1} 2$ & \\
\hline Mimosaceae & Enterolobium cyclocarpum (Jacq.) Griseb. ${ }^{1}$ & \\
\hline Mimosaceae & Inga vera Willd. ${ }^{1}$ & Carbonero \\
\hline Mimosaceae & Pithecellobium dulce (Roxb.) Benth. ${ }^{1 y 2}$ & Trupillo \\
\hline Mimosaceae & Samanea saman (Jacq.) Merr ${ }^{1}$ y 2 & Campano \\
\hline Moraceae & Batocarpus costaricensis Standl. \& L.O. Williams ${ }^{1}$ & Olla de mono \\
\hline Moraceae & Ficus americana Aubl. ${ }^{1}$ & Caoba \\
\hline Moraceae & Ficus cuatrecasana Dugand ${ }^{1}$ & Hobo macho \\
\hline Moraceae & Ficus nymphaeifolia Mill. ${ }^{1}$ & Lobo \\
\hline Myrsinaceae & Maclura tinctoria (L.) D. Don ex Steud. ${ }^{1}$ & Fruta pan \\
\hline Myrtaceae & Ardisia guianensis (Aubl.) Mez ${ }^{1}$ & Higuito \\
\hline Nyctaginaceae & Neea amplifolia Donn. Sm. ${ }^{1}$ & Ficus blanco \\
\hline Phyllanthaceae & Margaritaria nobilis L. f. $^{1}$ & \\
\hline Piperaceae & Piper marginatum Jacq. ${ }^{1}$ & Palo de mora \\
\hline Piperaceae & Piper pseudofuligineum C.DC. ${ }^{1}$ & \\
\hline Polygonaceae & Coccoloba nutans Kunth ${ }^{1}$ & Sembradera \\
\hline Polygonaceae & Coccoloba uvifera (L.) L. ${ }^{1}$ & Gajo de uvas \\
\hline Rubiaceae & Alibertia edulis (Rich.) A. Rich. ex DC. ${ }^{1}$ & \\
\hline Rubiaceae & Chione venosa (Sw.) Urb. ${ }^{1}$ & Uvero \\
\hline Rubiaceae & Coutarea hexandra (Jacq.) K. Schum. ${ }^{1}$ & Santa María \\
\hline Rubiaceae & Genipa americana $\mathrm{L}^{1}$ & \\
\hline Rubiaceae & Randia formosa (Jacq.) K Schum. ${ }^{1}$ & \\
\hline Rutaceae & Swinglea glutinosa (Blanco) Merr. ${ }^{1}$ & \\
\hline Rutaceae & Zanthoxylum caribaeum Lam. $^{1}$ & \\
\hline Salicaceae & Casearia aculeata Jacq. ${ }^{1}$ & Varita blanca \\
\hline Salicaceae & Xylosma intermedia (Seem.) Triana \& Planch. ${ }^{1}$ & Pigiguey \\
\hline Sapindaceae & Allophylus edulis (A. St.-Hil., A. Juss. \& Cambess.) Radlk. ${ }^{1}$ & Jagua \\
\hline Sapindaceae & Matayba scrobiculata Radlk. $^{1}$ & \\
\hline Simaroubaceae & Quassia amara L. 1 y 2 & \\
\hline Solanaceae & Cestrum alternifolium (Jacq.) O.E. Schulz ${ }^{1}$ & María Angola \\
\hline Theophrastaceae & Bonellia frutescens (Mill.) B.Ståhl \& Källersjö ${ }^{1}$ & Barbasco \\
\hline Theophrastaceae & Clavija spinosa (Vell.) Mez. ${ }^{1}$ & Guacharaco \\
\hline Ulmaceae & Trema micrantha (L.) Blume ${ }^{1}$ & Guácimo \\
\hline Urticaceae & Cecropia peltata L. ${ }^{1}$ & Naranjuelo \\
\hline Verbenaceae & Lantana camara L. 1 y 2 & Venturosa \\
\hline Verbenaceae & Vitex orinocensis Kunth ${ }^{1}$ & \\
\hline
\end{tabular}



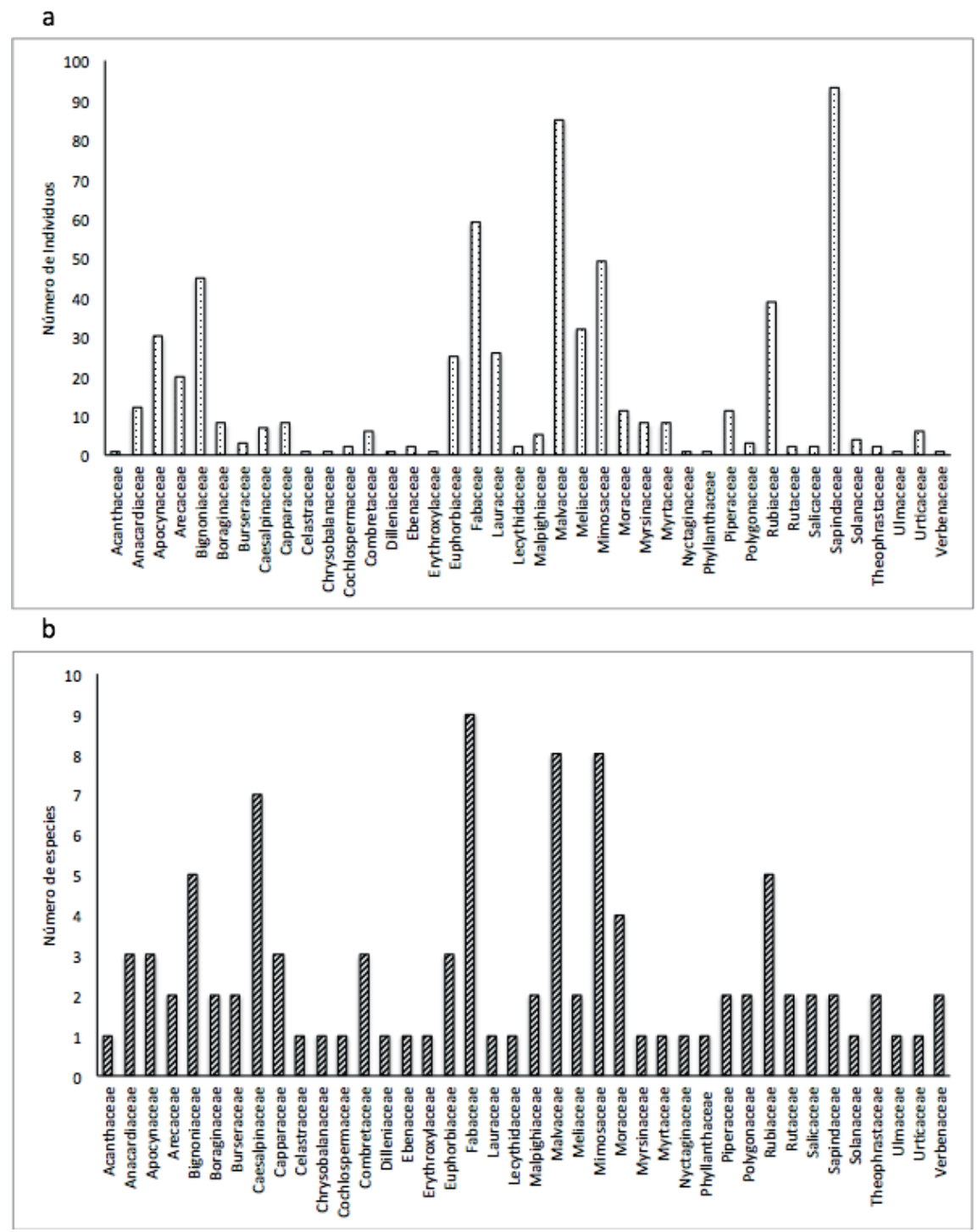

Figura 2. (a) Abundancia y (b) riqueza de especies por familia de plantas leñosas en la Reserva Sanguaré-Sucre (Colombia).

De los 89 géneros identificados el más rico es Machaerium con cuatro especies, seguido por Ficus, representados por tres especies. Con respecto a las abundancias, las especies más importantes fueron M. scrobiculata, S. apetala y G. ulmifolia, las cuales estuvieron presentes en todos los transectos, seguida por T. rosea y A. niopoides de la comunidad arbórea y arbustiva (figura 3a). Asimismo, fueron dominantes A. butyracea, seguida de A. caribea, G. ulmifolia, $N$. membranacea, E. cyclocarpum y S. mombin (figura $3 b)$. Finalmente, las especies más frecuentes fueron S. apetala, A. butyracea, T. cymosa, G. ulmifolia, y T. hirta (figura 3c).

La mayor parte de los géneros presentaron un valor de importancia muy bajo, sin embargo, fue posible establecer con valores relativamente altos a Attalea con el 10,2\%, Matayba 6,4\%, Guazuma $6,38 \%$ y Albizia con 6,26\%. En el mismo orden, las especies más importantes del bosque fueron: $A$. butyracea con el 10,2\%, seguida de M. scrobiculata con el 6,4\%; G. ulmifolia con el 6,38\%; A. niopoides con $6,3 \%$ y $S$. apetala con el $5,8 \%$. Al sumar el valor de importancia de todas las especies se obtuvo un valor de 2,97, cuyo porcentaje es del $99 \%$. Es importante tener en cuenta que el índice calculado es aproximado a 3, lo cual corresponde al valor máximo teórico para una comunidad vegetal.

La familia Fabaceae presentó el mayor valor de índice de importancia (19,5\%), le siguen Malvaceae $(12,3 \%)$ y Arecaceae $(10,2 \%)$ con una especie y varios individuos que suman un elevado valor de área basal. Sapindaceae $(6,8 \%)$ y Bignoniaceae $(5,1 \%)$ 

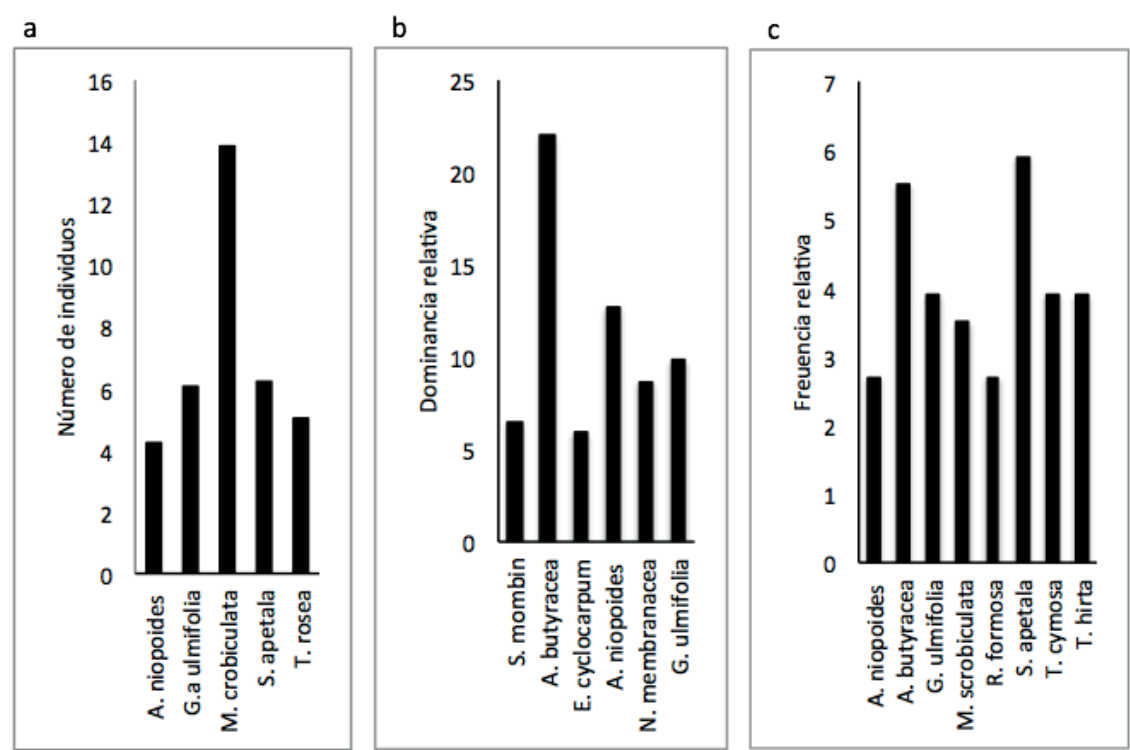

Figura 3. (a) Especies más abundantes, (b) dominantes y (c) frecuentes en la Reserva Sanguaré-Sucre (Colombia).

también presentaron valores relevantes; empero, la abundancia con respecto al número de especies por área basal tuvo un comportamiento inverso. Es decir, en el caso de Sapindaceae, esta es abundante pero en su área basal es menor; mientras que Bignoniaceae obtuvo una baja abundancia, pero un área basal mayor que Sapindaceae.

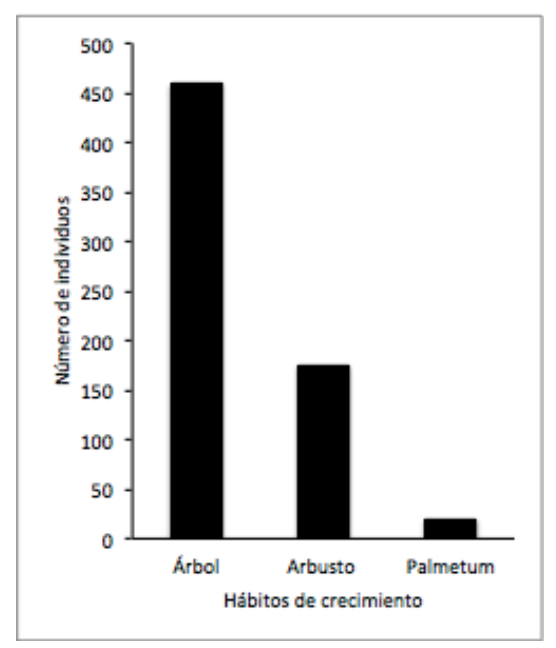

Figura 4. Abundancia de los hábitos de crecimiento, árbol, arbusto y palmetum en el bosque seco en la Reserva Sanguaré-Sucre (Colombia).

\subsection{Estructura de la vegetación}

El área muestreada del bosque de la Reserva Natural Sanguaré tiene una cobertura determinada por cuatros estratos como son el arbóreo, palmetos, arbustivo y herbáceo (figura 4). Un total de ocho clases de alturas fueron generadas, siendo la clase 2 la que in- cluyó el mayor número de individuos, seguida por la clase de 3. Esta última clase también presentó el mayor número de especies, después de la clase 4 y 2 respectivamente (figura 5). Un resultado importante es que $A$. niopoides se encontraba en todas las clases de altura junto con T. rosea, T. hirta y S. apetada (tabla 2).

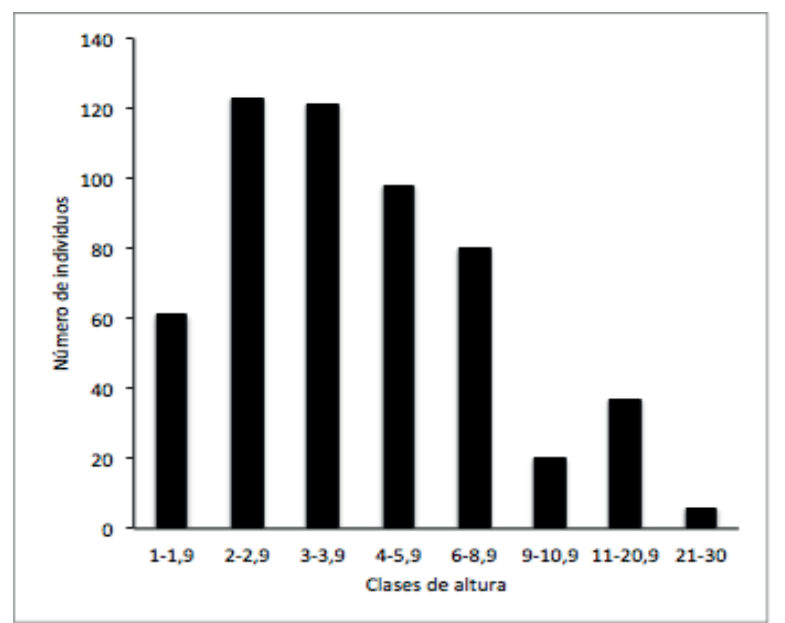

Figura 5. Abundancia por clases de alturas en el bosque de la Reserva Sanguaré-Sucre (Colombia).

De acuerdo con el hábito de crecimiento, los árboles presentaron la cobertura dominante con los $61 \%$, seguidos por el $38 \%$ de arbustos y solo el $1 \%$ de palmas. Con respecto a la altura promedio, en su mayoría las especies no sobrepasaron los $15 \mathrm{~m}$, siendo el taxón con mayor altura Albizia niopoides con 30 metros (tabla 2). En cuanto a los arbustos, las alturas comprendidas en el rango 2 a $4 \mathrm{~m}$, fueron registradas para los individuos de Randia formosa. 
Tabla 2. Especies más importantes en las diferentes clases de alturas (metros) en el bosque seco Sanguaré-Sucre (Colombia): 1-1,9 (1); 2-2,9 (2); 3-3,9 (3); 4-5,9 (4); 6-8,9 (5); 9-10,9 (6); 11-20,9 (7); 21-30 (8).

\begin{tabular}{|c|c|c|}
\hline Albizia niopoides $^{1,2,3,4,5,6,7 y 8}$ & Enterolobium ciclocarpum ${ }^{4,5,6 y 7}$ & Randia Formosa ${ }^{2}$ \\
\hline Anacardiun excelsum ${ }^{4}$ & Genipa americana ${ }^{3}$ & Sapium glandulosum ${ }^{2 y 3}$ \\
\hline Andira inermis $1,2,3,4,5 y 6$ & Guazuma ulmifolia ${ }^{1,2,3,4,5,6 y 7}$ & Sapium glandulosum ${ }^{3}$ \\
\hline Aphelandra scabra ${ }^{2}$ & Guazuma ulmifolia ${ }^{3,4,5,6 y 7}$ & Spondias mombin ${ }^{4,5,6 y 7}$ \\
\hline Astronium graveolens $s^{2,3 y 4}$ & $H$ y menae courbaril ${ }^{5}$ & Spondias mombin $5,6 y 7$ \\
\hline Attalea but y racea $a^{3,4,5 y 6}$ & Leguncularia racemosa ${ }^{5}$ & Sterculia apetala $1,2,3,4,5,6,7 y 8$ \\
\hline Bursera simaruba ${ }^{6}$ & Machaerium capote ${ }^{3}$ & Tabebuia bilbergii ${ }^{2 y 3}$ \\
\hline Cecropia peltata ${ }^{5,6,7 y 8}$ & Maclura tinctoria ${ }^{3}$ & Tabebuia rosea $a^{1,2,3,45 y 6}$ \\
\hline Chrysobalanus icaco ${ }^{4}$ & Matayba scrobiculata ${ }^{1,2,3,4,5,6,7 y 8}$ & Trichilia hirta ${ }^{1,2,3,4,5,6 y 7}$ \\
\hline Copaiferan officinalis ${ }^{1}$ & Piper arboreum ${ }^{1 y 2}$ & Trichilia pallida $a^{455}$ \\
\hline Cordia collococca $a^{4,5,6 y 7}$ & Piper marginatum ${ }^{3}$ & Xylosma intermedium ${ }^{3}$ \\
\hline${\text { Crescentia } \text { cujete }^{2,3 y 4}}^{2,3}$ & Quadrella odoratissima ${ }^{5}$ & \\
\hline
\end{tabular}

\section{Discusión}

\subsection{Riqueza y composición florística}

Los bosques secos de Sanguaré presentan una riqueza similar a las de otras localidades de bosques secos en Colombia [3]. En términos generales la riqueza es menor a la hallada en los bosques húmedos, lo cual es una tendencia general de estos bosques [3]. Un total de 82 especies de plantas leñosas fueron identificadas en este estudio, consecuente con los resultados de otras localidades con un área de muestreo de similar tamaño [41, 42]. Las familias más importantes fueron Fabaceae, Malvaceae, Bignoniaceae y Rubiaceae, lo que coincide con otros estudios de bosque seco [41, 42].

Al realizar una comparación entre la riqueza observada en Sanguaré con otros trabajos florísticos reportados en otras localidades, se pudo determinar que el análisis realizado por GEMA (84 familias y 320 especies) en los bosques de los Navas y el bosque de la Estación Primates [43], la riqueza es mayor con respecto a familias y especies. Empero, los datos obtenidos en Sanguaré son contrastantes con otras localidades. En el presente estudio se observa un mayor número de familias pero menos especies a las encontradas en Colosó (Sucre) y Los Colorados en Bolívar (4 y 41 familias, 113 y 121 especies, respectivamente) por Gentry [21]. Asimismo, este patrón es observado al comparar Sanguaré con San Sebastián, Magdalena (42 familias y 155 especies) [44]. Estos resultados pueden ser el reflejo de las condiciones del bosque, Sanguaré está en estado de regeneración natural restauración, mientras que San Sebastián, Primates en Colosó y los Colorados son bosques relictuales, en los cuales sus comunidades pueden estar más conservadas [45].
Por otra parte, también es notable que el número de familias y especies en Sanguaré es mayor en relación a otros ecosistemas de bosque seco. En Tierra Bomba, según Mendoza [5] presentó 26 familias y 56 especies; para Galerazamba y Tayrona [21], se reportaron 26 familias y 56 especies, en el Cerro Tasajero Carrillo, et al. [41] reporta 34 familias y 79 especies; mientras que en la Reserva Forestal Río Blanco (Manizales), Sanin, et al. [46] identifican 37 familias y 73 especies; y en la Finca Forestal Monterrey registran 24 familias y 55 especies [5]. Varios estudios han reportado que los bosques al encontrarse en estado de regeneración natural, son más propensos a ser colonizados por diferentes especies que evitan la competencia por la luz, de tal forma que se puede observar mayor diversidad que la establecida en un bosque maduro [24]. En este sentido, la alta riqueza observada en Sanguaré, puede ser el resultado actual del proceso de regeneración ecológica al cual ha sido sometido, lo que ofrece mayores bases para proponer los bosques de esta Reserva como bosques secundarios.

Con respecto a la composición general de la flora en Sanguaré, la estructura de los taxones es similar a la propuesta por Gentry [21] para el bosque seco tropical, dentro de la cual los grupos más importantes son Leguminosae y Bignoniaceae, seguidas por Rubiaceae, Sapindaceae, Euphorbiaceae, Flacourtiaceae y Capparaceae. Sin embargo, en Sanguaré solo fue dominante Fabaceae, comprendida por los géneros Albizia sp., Calliandra sp., Copaifera sp., Inga sp., Machaerium sp.,entre otros. Otros grupos que también son importantes, pero menos abundantes y ricos a lo descrito por Gentry (20) fueron Rubiaceae, Ephorbiaceae, Moraceae y Bignoniaceae, aunque 
con bajo número de especies en comparación con Fabaceae [7, 11].

Rubiaceae, es una familia fuertemente diversa en los bosques húmedos y andinos [47], que se ha establecido con relativa importancia en los bosques secos; en Sanguaré por ejemplo, estuvo representada por ocho especies. Datos similares fueron observados por Marulanda et al. [44] quien encontró la más alta representación de esta familia con 10 especies en los bosques secos en Magdalena, Colombia. Por su parte, Mendoza [5] en la costa Caribe presenta a Los Colorados como el sitio que posee mayor representación de Rubiaceae con seis especies, seguido por Neguanje con cinco y la Finca Forestal Monterrey y Tierra Bomba con sólo dos representantes. Así mismo, GEMA [15] y Mendoza [5] en el valle seco interandino del río Magdalena, reportan en Bremen cuatro especies de Rubiaceae; mientras que en Santo Tomás y Cardonal solo tres y dos especies, respectivamente. Estos datos soportan lo propuesto por Mendoza [5] cuando sugiere que en los bosques secos en Colombia, esta familia es una de la más representada y que es mucho más importante en los bosques de la región del Caribe colombiano que en los del valle interandino del río Magdalena. Con base en lo reportado, Sanguaré presenta la mayor riqueza de Rubiaceae, entre los bosques secos de Colombia.

Por otra parte, Bignoniaceae ha sido catalogada como uno de los grupos más relevantes en el bosque seco, sin embargo para Sanguaré solo se hallaron cuatros especies (tabla 1). Este resultado es una consecuencia del tipo de muestreo realizado, ya que solo fueron recolectados y censados los individuos leñosos. Bignoniaceae es una familia ampliamente diversa en el Neotropico; sin embargo, parte de su diversidad se conforma por taxones de habito lianescente [48], de tal forma que al no censar las lianas en Sanguaré muy posiblemente se perdió parte de información sobre la riqueza de este grupo. No obstante, aunque las lianas no fueron muestreadas, si se pudo establecer el registro de 10 individuos de Handroanthus billbergii especie poco representada en los bosques secos [5].

Para el caso de la familia Euphorbiaceae, Murillo [49], encuentra que este taxón está ampliamente distribuido por todas las regiones naturales de Colombia. Además, señala que la región Caribe ocupa el tercer lugar en cuanto a especies propias de esta familia con 114 especies, por lo tanto, su alta representación en el bosque de Sanguaré no es un dato atípico a lo sugerido por Murillo. Otro dato importante es la presencia en altos valores de Piperaceae, la cual no es incluida normalmente en los reportes realizados en otros ecosistemas de bosque seco. Callejas [com. pers.], plantea que esta familia es muy común en el Neotrópico, y muy frecuente en áreas perturbadas y sitios expuestos del bosque húmedo, lo cual se encuentra en concordancia a las especies halladas en Sanguaré en lugares en restauración como los borde de bosque y bosques secundarios.

Por otra parte, el valor de importancia de las familias y especies, tenemos que Fabaceae fue el taxón más importante con alrededor de 23 especies, representando el $23,1 \%$, del total de taxones en el Sanguaré. La presencia de la Arecaceae, fue algo completamente atípico a lo observado en el bosque seco, principalmente por la dominancia de Attalea butyracea como el segundo grupo dominante. Este resultado puede ser producto de la cercanía de los puntos al mar o a factores climáticos (climas poco pluviales con vientos húmedos predominantes) y edáficos (suelos ácidos y bien drenados) que favorecen el crecimiento de esta especie. Otras especies con un valor de importancia alto fueron G. ulmifolia, T. rosea, A. niopoides, $M$. scrobiculata, entre otras. Este resultado es comparable con los resultados de Carrillo-Fajardo, et al. [41] donde se establece que G. ulmifolia es el segundo grupo más importante en Cerro Tasajero. Estos datos pueden ser el resultado de la rápida dispersión de sus semillas por especies de mamíferos silvestres y de ganado vacuno, lo cual favorece la regeneración ecológica de una zona que se encuentra en estado de sucesión, como lo es la Reserva Sanguaré.

\subsection{Estructura de la vegetación}

Con respecto al hábito de crecimiento de las especies observadas en el bosque de la Reserva Natural Sanguaré, los datos son similares en los hábitos arbóreo, arbustivo y palmeto reportados para San Sebastián por Marulanda et al. [44]. Es notorio que la cercanía al litoral marino ha jugado un papel importante en la configuración de la flora en Sanguaré, lo cual es claro por la alta abundancia del palmeto en esta zona. Este resultado es contrastante con otras localidades de bosque seco donde los árboles 
son abundantes seguidos de los arbustos, pero las palmas ocupan una posición menos relevante (Colorados y Neguanje por Mendoza [5] y Serró Tasajero por Carrillo et al. [41]). No obstante, la abundancia del palmetum solo es un dato atípico ya que en términos generales la presencia de 59 especies de habito arbóreo y 35 arbustivas evidencian que los hábitos observados en la reserva son similares a los determinados para el bosque seco [21].

Las alturas observadas en este trabajo, muestran claramente que las especies no sobrepasan una altura de $15 \mathrm{~m}$. La mayor parte de los individuos presentan un intervalo de altura entre los 4-6 metros. Estos datos muestran claramente el estado temprano de regeneración en el que se encuentra el bosque, lo cual nos permite tipificarlo como bosque secundario, con la presencia de algunos árboles que permanecen luego de los eventos de desforestación del bosque prístino [30]. Los valores promedio de altura observados en este estudio, son comparables con los analizados por Dechner y Diazgranados [50], el cual alcanzó una altura promedio de 20,1 m para la Sierra Nevada de Santa Marta. Sin embargo, es menor al compararlo con el bosque Estación Primates, el cual presenta una altura que varía entre 25 y $30 \mathrm{~m}$ [43] o lo descrito por Lozano [12] para el Parque Nacional Natural Tayrona, que alcanza con frecuencia alturas de $30 \mathrm{~m}$.

La causa de estas variación puede ser acuñada al poco desarrollo de los individuos en algunas zonas del bosque de la reserva, el cual tuvo una fuerte intervención durante los años de 1943 a 1974 (Ruiz, en prep.). En el bosque hay áreas con elementos en regeneración, lo cual es soportado por la anotaciones de Fajardo et al. [51] quien explica que un bosque con baja altura del dosel es un bosque que ha tenido un grado de intervención originando así la perdida de muchos elementos del estrato arbóreo. Asimismo, la existencia de este tipo de bosque se debe por un lado a los límites climáticos de precipitación y biotemperatura [52] y por el otro, la condición determinada por la intervención y destrucción al cual fue sometido [30].

La recuperación que ha tenido dicho bosque, a partir de la decisión de los actuales propietarios en convertir dicha zona en una reserva natural, ha representado una cobertura vegetal cuya extensión es de 133 ha, cuando en el año 1992 llegó ha ser de 75 ha.
Con el cambio del paisaje se alteró la estructura y composición del bosque pristino, para dar paso a una estructura y composición propia de los pastizales y cultivos que más tarde cambió de propósito, de tal forma que, que a partir de 1994 se proyectó implementar una zona de reserva permitiendo la expresión de la sucesión natural la cual ha determinado el establecimiento de una cobertura vegetal constituida por plantas herbáceas, arbustivas, arbórea y palmeto, en donde se presentó la dominancia de la familia Leguminosa y la presencia de árboles relictuales del bosque primario entre ellos: Albizia caribea, Enterolobium cyclocarpum, Swietenia macrophylla; el hábito de crecimiento arbóreo fue seguido por el de arbustos y palmetos.

\section{Conclusiones}

Los resultados obtenidos en este estudio, son la primera contribución hacia el conocimiento de la flora en la región del Golfo de Morrosquillo, pudiendo establecer que el bosque seco tropical se expande por fuera de los Montes de María. La riqueza observada en este estudio es similar a la establecida en otras localidades con presencia de bosque seco en Colombia. Las familias Fabaceae, Malvaceae y Rubiaceae son tres grupos de gran importancia por el alto número de especies e individuos. Un resultado importante en este estudio es el reporte de $H$. bilbergii, taxón considerado como endémico de los bosques secos del Caribe.

La composición florística de Sanguaré muestra una alta riqueza con referente a otros fragmentos de bosque seco tropical, teniendo en cuenta que este ecosistema se encuentra aún en estado de sucesión, luego del fuerte impacto antrópico que sufrió hace más de 50 años. En relación a los grupos más dominantes se incluyen a Attalea y Matayba, como los géneros más importantes en términos de abundancia y DAP. Cabe resaltar que la alta representación de Attalea butyracea, es un dato atípico en el bosque seco, ya que esta especie no es dominante en estos ecosistemas.

Los árboles son dominantes en los bosques de Sanguaré, no obstante, la altura promedio es de 2 a 9 metros de altura, siendo los $15 \mathrm{~m}$ la altura promedio observada, lo cual es una consecuencia de estado temprano de sucesión del sitio. Empero, fue notable que especies como Albizia niopoides presentaron 
más de $30 \mathrm{~m}$ de altura; mientras que taxones como Cecropia peltata, Cordia collococca, Enterolobium ciclocarpum, Guazuma ulmifolia, Matayba scrobiculata, Spondias mombin, Sterculia apetala y Trichilia hirta sobrepasaron los 20 metros. Estas especies dan una pequeña muestra de los taxones que conformaban estos bosques antes del proceso de deforestación al cual fueron sometidos.

La proyección del área para un proyecto de Reserva Natural (1994) permitió el proceso de sucesión natural con el inicio de la recuperación de la flora que tiene curso en lo que hoy se conoce como la Reserva Natural de la Sociedad Civil Sanguaré, la cual es figura jurídica a partir del 22 de agosto del 2002, años en los que se ha recuperado de la vegetación propia del bosque seco con una cobertura de 133 ha, donde el $9 \%$ corresponde a la cobertura que existió en antaño en la zona de la actual reserva.

Se recomienda en un futuro evaluar la diversidad y realizar una comparación entre los dos puntos muestreados, con el fin de establecer afinidades o diferencias entre estas, así como entender si la diversidad aumenta o se mantiene sobre el tiempo. Teniendo en cuenta que son dos áreas boscosas que aún se encuentran en un proceso de sucesión activo.

Finalmente, se recomienda ampliar los estudios de diversidad en el Caribe, ya que son poco los realizados, teniendo en cuenta el tamaño de ciertas áreas boscosas como los Montes de María. No obstante, es de gran importancia expandir los muestreos hacia otras subregiones del departamento con el fin de identificar todos los fragmentos de bosques seco, y así poder implementar estrategias de conservación y áreas prioritarias para tal fin.

\section{Agradecimientos}

Al Señor Álvaro Hernán Roldán administrador de la Reserva Natural Sanguaré y su esposa Diana, por haber creído en nosotros, por brindarnos toda su colaboración, respaldo, consejos y confianza. A los trabajadores de Sanguaré, Margarita Cali, Vivian Cali. A Felipe Cardona director general del Herbario de la Universidad de Antioquia, Francisco Roldán curador del HUA y a Ricardo Callejas docentes de la Universidad de Antioquia. Los profesores Santiago Ruiz Pérez, Gustavo Barros y Hernando Gómez (Universidad de Sucre) por sus aportes al manuscrito.

\section{Referencias}

[1] P. G. Murphy y A. E. Lugo, "Ecology of tropical dry forest", Annual Review of Ecology and Systematics, vol. 17, pp. 67-88, 1986.

[2] R. T. Pennington, M. Lavin, y A. OliveiraFilho, "Woody plant diversity, evolution, and ecology in the tropics: Perspectives from seasonally dry tropical forests", Annual Review of Ecology, Evolution, and Systematics, vol. 40, pp. 437-457, 2009.

[3] H. García, G. Corzo, P. Isaac, y A. Etter, "Distribución y estado actual de los remanentes del bioma de bosque seco tropical en Colombia: Insumos para su conservación", in El bosque seco tropical en Colombia. vol. 90, C. Pizano and H. García, Eds., Bogotá, D.C: Instituto de Investigación de Recursos Biológicos, Alexander von Humboldt, 2014, pp. 228-251.

[4] C. A. Portillo-Quintero y G. A. SánchezAzofeifa, "Extent and conservation of tropical dry forests in the Americas", Biological Conservation, vol. 143, pp. 144-155, 2010.

[5] H. Mendoza-C., "Estructura y riqueza florística del bosque seco tropical en la región Caribe y el valle del río Magdalena, Colombia", Caldasia, vol. 21, pp. 70-94, 1999.

[6] C. Pizano, R. González, M. Gonzales, R. Castro-Lima, N. Rodríguez, y A. Idárraga, et al., "Plantas de los bosques secos de Colombia", in El bosque seco tropical en Colombia, C. Pizano and H. García, Eds., Bogotá, DC: Instituto de Investigaciones Alexander von Humboldt, 2014, pp. 37-48.

[7] H. Mendoza, "Estructura y riqueza florística del bosque seco tropical en la región Caribe y el valle del río Magdalena, Colombia", Caldasia, vol. 21, pp. 70-94, 1999.

[8] W. Rieger, Vegetations kundliche untersuchungen auf Guajira-Halbinsel (NordostKolumbien). Geographischen Institut der Justus Liebig - Universitat GieBen: Alemania, 1976.

[9] A. M. Sudgen y R. J. Robins, "Aspects of the ecology of vascular epiphytes in Colombia could forest, I. the distribution of the epiphitic flora", Biotropica, vol. 11, pp. 175-188, 1979.

[10] A. M. Sugden y E. Forero, "Catalogo de las plantas vasculares de la Guajira con comentarios sobre la vegetación de la Serranía de la Ma- 
cuira",Colombia Geográfica (Revista IGAC), vol. 10, pp. 23-75, 1982.

[11] E. Carbonó-De la Hoz y H. García, "La vegetación terrestre en la ensenada de Neguanje, parque nacional natural Tayrona (Magdalena, Colombia)", Caldasia, vol. 32, pp. 235-256, 2010.

[12] G. Lozano, "Comunidades vegetales del flanco norte del cerro "El Cielo" y la flora vascular del Parque Nacional Tayrona (Magdalena, Colombia)", in La Sierra Nevada de Santa Marta (Colombia) Transecto Buritaca-la Cumbre. En estudios de Ecosistemas Tropandinos, v. d. Hammen and Ruíz, Eds., Berlín: J Cramer, 1984, pp. 407-422.

[13] G. Lozano-C., "Comparación Florística del Parque Nacional Natural Tayrona, La Guajira y La Macuira-Colombia y los Médanos de CoroVenezuela", Mutisia, vol. 67, pp. 1-26, 1986.

[14] H. Cuadros, "Vegetación caribeña. En Caribe Colombia", FEN, Colombia, Bogotá, pp. 67-84, 1996.

[15] I. Instituto Alexander von Humboldt, Caracterización ecológica de cuatro remanentes de bosque seco de la región Caribe colombiana. Villa de Leyva: Grupo de Exploraciones y Monitoreo Ambiental GEMA, 1997.

[16] C. Pizano y H. García, El bosque seco tropical en Colombia. Bogotá, D.C., Colombia: Instituto de Investigación de Recursos Biológicos Alexander von Humboldt, 2014.

[17] E. Barrios Paternina, J. Mercado Gómez, "Plantas útiles del corregimiento Santa Inés y vereda San Felipe-San Marcos (Sucre, Colombia)", Ciencia en Desarrollo, vol. 5, no. 2, pp. 131144, 2014.

[18] A. Cuervo, C. Barbosa, y J. De la Ossa, "Aspectos ecológicos y etológicos de primates con énfasis en Alouatta seniculus (Cebidae), de la región de Colosó, Serranía de San Jacinto (Sucre) Costa Norte de Colombia", Caldasia, vol. 14, pp. 68-70, 1986.

[19] S. Galván-Guevara, M. I. Sierra, f. H. Gómez, V. J. De la Ossa, y A. Fajardo-Patiño, "Biodiversidad en el área de influencia de la estación primates de Colosó, Sucre, Colombia", Recia, vol. 1, 2009.
[20] R. Patiño y O. Rangel, "Estudio de caso. La vegetación boscosa en la estación de primates Coloso - Sucre", in Colombia diversidad biótica XI. Patrones de la estructura y de la riqueza de la vegetación en Colombia, O. Rangel-Ch, Ed., Bogotá: Universidad Nacional de Colombia (Sede Bogotá) Facultad de Ciencias Instituto de Ciencias Naturales, 2011, pp. 253-268.

[21] A. Gentry, "Diversity and floristic composition of neotropical dry forests", in Seasonally Dry Tropical Forests. vol. 85, S. H. Bullock, H. A. Mooney, and E. Medina, Eds., Cambridge: Cambridge University Press, 1995, pp. 146194.

[22] M. A. Sampedro, F. H. Gómez, y D. G. Ballut, "Estado de la vegetación en localidades abandonadas por "desplazamiento", en los montes de María Sucre, Colombia, Recia, vol. 6, pp. 184-193, 2014.

[23] A. Sampedro, A. Álvarez, L. M. Domínguez, y I. Herrera, "Especies promisorias para el ecoturismo en "Campo Aventura Roca Madre", Toluviejo-Sucre, Colombia", Rev. MVZ Córdoba, vol. 18, pp. 3387-3398, 2013.

[24] D. Olascuaga y J. Mercado-Gómez, “Análisis de la vegetación sucesional en un fragmento de bosque seco tropical en Toluviejo-Sucre (Colombia)", Colombia Forestal vol. 19, pp. 23-40, 2016.

[25] R. Salcedo y A. Santos, "Etnobiología de los montes de María sector nororiental 'vereda La Piche', municipio de Toluviejo-Sucre”, Departamento de Biología, Trabajo de grado Biología, Universidad de Sucre, Sincelejo, Sucre, 2011.

[26] Á. E. Álvarez Puentes, L. M. Domínguez, y I. M. Herrera Méndez, "Aplicación de técnicas ecológicas para el desarrollo del ecoturismo y la conservación de la diversidad biología en campo aventura Roca madre, Toluviejo Sucre", Departamento de Biología, Trabajo de grado Biología, Universidad de Sucre, Sincelejo, 2010.

[27] D. Olascuaga, "Análisis de la vegetación sucesional en un remanente de bosque seco tropical en Toluviejo-Sucre (Colombia)", Departamento de Biología, Trabajo de grado Biología, Universidad de Sucre, Sincelejo-Sucre, 2014. 
[28] Cauzado-Zapa, "Análisis fitosociológico del ecosistema del manglar del parque regional natural boca guacamaya, departamento de sucre ", Departamento de Biología, Trabajo de grado Biología, Universidad de Sucre, SincelejoSucre, 2010.

[29] D. Angarita-Hernández, S. D, y J. MercadoGómez, "Nuevos registro corológicos para $\mathrm{Su}-$ cre (Sanguaré - Colombia)", Recia, vol. 6, pp. 360-373, 2014.

[30] J. Huertas, "Implementación de un sistema de información geográfica en la reserva Natural de la Sociedad Civil Sanguaré, San Onofre Colombia", Documento de trabajo No. 1, Universidad de Antioquia, Colombia, 2005.

[31] D. Angarita-Hernández y D. San Martín- Sierra, "Estudio multitemporal en el remanente de bosque seco tropical e inventario de plantas leñosas en la reserva natural de la sociedad civil sanguaré localizada en el municipio de San Onofre - Sucre.”, Departamento de Biología, Trabajo de grado Biología, Universidad de Sucre, Sincelejo-Sucre, 2010.

[32] A. Gentry, "Patterns of neotropical plant species diversity", Evolution biology, vol. 15, pp. 1-84, 1882.

[33] C. ISA- JAUM, "Programa de biodiversidad. Propuesta metodológica de parcelas normalizadas para los inventarios de vegetación”, 2004.

[34] M. B. Gargiullo, B. L. Magnuson, y L. D. Kimball, A field guide to plants of Costa Rica. New York: Oxford University Press, 2008.

[35] A. Gentry, A Field Guide to the Families and Genera of Woody Plants of Northwest South America: (Colombia, Ecuador, Peru : With Supplementary Notes on herbaceus taxa. Washinton, DC: Conservations international 1993.

[36] B. J. Enquist y J. J. Sullivan, Vegetative key and descriptions of tree species of the tropical dry forests of upland Sector Santa Rosa, Área de Conservación Guanacaste, Costa Rica. Auckland: Manaaki Whenua - Landcare Research, Private Bag, 2001.

[37] B. Boyle, N. Hopkins, Z. Lu, J. A. Raygoza Garay, D. Mozzherin, y T. Rees, et al., "The taxonomic name resolution service: an online tool for automated standardization of plant names", BMC Bioinformatics, vol. 14, p. 16, 2013.

[38] APG III, "An update of the Angiosperm Phylogeny Group classification for the orders and families of flowering plants: APG III", Botanical journal of the Linnean Society, vol. 161, pp. 105-121, 2009.

[39] O. Rangel-Ch y A. Velásquez, "Métodos de estudio de la vegetación", in Colombia diversidad biótica II. Tipos de vegetación en Colombia, O. Rangel-Ch, P. D. Lowy-C, and M. Aguilar-P, Eds., Bogotá: Editorial Guadalupe Ltda, 1997, pp. 59-82.

[40] T. van der Hammen y O. Rangel-Ch, "Recuento histórico- tareas futuras", in Colombia diversidad biótica II. Tipos de vegetación en Colombia, O. Rangel-Ch, P. D. Lowy-C, and M. Aguilar-P, Eds., ed Bogotá: Editorial Guadalupe Ltda, 1997, pp. 17-58.

[41] M. Carrillo-Fajardo, O. Rivera-Díaz, y R. Sánchez-Montaño, "Caracterización florística y estructural del bosque seco tropical del Cerro Tasajero, San José de Cúcuta (Norte de Santander), Colombia", Actualidades biológicas, vol. 29, pp. 55-73, 2007.

[42] A. M. Torres, J. B. Adarve, M. Cárdenas, J. A. Vargas, V. Londoño, y K. Rivera, et al., "Dinámica sucesional de un fragmento de bosque seco tropical del Valle del Cauca, Colombia", Biota Colombiana, vol. 13, pp. 40-69, 2012.

[43] A. Acosta, "Caracterización de la biodiversidad y de los Sistemas de uso en áreas de influencia de la corporación autónoma regional de Sucre", documento No 1, Colombia., 2009.

[44] L. O. Marulanda, A. Uribe, P. Velásquez, M. A. Montoya, A. Idárraga, y M. C. López, et al., "Estructura y composición de la vegetación de un fragmento de bosque seco en San Sebastián, Magdalena (Colombia). I. Composición de plantas vasculares", Actualidades biológicas, vol. 25, pp. 17-30, 2003.

[45] H. E. Ireland, G. C. Kite, N. C. Veitch, M. W. Chase, B. Schrire, y M. Lavin, et al., "Biogeographical, ecological and morphological structure in a phylogenetic analysis of Ateleia (Swartzieae, Fabaceae) derived from combined molecular, morphological and chemical data", 
Botanical Journal of the Linnean Society, vol. 162, pp. 39-53, 2010.

[46] D. Sanín, J. C. Mancera-Santa, N. CastañoRubiano, N. F. Álzate-Q, G. González-O, y L. M. Álvarez-M, "Catálogo preliminar de las plantas vasculares de la Reserva Forestal protectora "Río Blanco" (Manizales, Caldas, Colombia)", Boletín Científico - Centro de Museos - Museo de Historia Natural, vol. 10, pp. 19-44, 2006.

[47] H. Mendoza, B. Ramírez, y L. C. Jiménez, $R u$ biaceae de Colombia. Guía ilustrada de géneros. Bogotá, Colombia: Instituto de Investigación de Recursos Biológicos Alexander von Humboldt, 2004.

[48] L. G. Lohmann, "Untangling the phylogeny of neotropical lianas (Bignonieae, Bignoniaceae)", American Journal of Botany, vol. 93, pp. 304-318. 2006.

[49] J. Murillo-A, C. Polanía, y A. León-P, "Los helechos y licófitos de la región del Guavio", Biota Colombiana, vol. 9, pp. 63-76, 2008.

[50] A. Dechner y M. Diazgranados, "Composición y estructura de la vegetación boscosa de la cuenca baja del Río San Salvador, vertiente norte de la Sierra Nevada de Santa Marta, Colombia", Universitas Scientiarum, vol. 12, pp. 99-124, 2007.

[51] L. Fajardo, V. González, J. M. Nassar, P. Lacabana, C. A. Portillo Q, y F. Carrasquel, et al., "Tropical Dry Forests of Venezuela: Characterization and Current Conservation Status 1", Biotropica, vol. 37, pp. 531-546, 2005.

[52] L. R. Holdridge, Ecología basada en zonas de vida. San José, Costa Rica: Instituto Interamericano de Ciencias Agrícolas, 1978. 